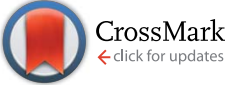

Cite this: RSC Adv., 2017, 7, 10831

\title{
Thermal behavior and molecular mobility in the glassy state of three anti-hypertensive pharmaceutical ingredients
}

\begin{abstract}
Joaquim J. Moura Ramos ${ }^{\star a}$ and Hermínio P. Diogo*b
The slow molecular mobility in the amorphous solid state of three active pharmaceutical drugs (valsartan, telmisartan and irbesartan) has been studied using differential scanning calorimetry (DSC) and the dielectric technique of thermally stimulated depolarization currents (TSDC). The thermal behavior of the three substances (melting, glass forming ability, glass stability) and the features of their amorphous solid state (glass transition, activation energy for the structural relaxation) were studied by DSC. The TSDC study of the mobility in the glassy state yielded the main kinetic features of the $\alpha$ - and of the secondary relaxations. According to the obtained results the three studied glass forming drugs behave as strong liquids and telmisartan is the one with the greatest dynamic fragility.
\end{abstract}

Received 8th January 2017

Accepted 3rd February 2017

DOI: $10.1039 / \mathrm{c} 7 \mathrm{ra00298j}$

rsc.li/rsc-advances

in more amorphous APIs helps to better understand the relationship between mobility and instability and to find the best processing and storage conditions to ensure greater stability. However, the vitreous state may not be the best formulation to market a drug, either because of instability problems, or because the increasingly high molecular complexity of the new drugs makes them less and less soluble in water even in the amorphous state, or because there are other ways of achieving greater bioavailability, or finally because drugs with a glass transition temperature below room temperature are not good candidates to be delivered in the pure amorphous solid form.

Therefore, different strategies to improve the solubility of poorly water-soluble compounds have been developed. One of them is to deliver the drug in the form of an amorphous solid dispersion (ASD). ${ }^{10,11}$ Although most APIs are not marketed in the form of pure amorphous solid, the study of molecular mobility in the pure amorphous remains important because the relative crystallization tendency of drugs from amorphous solid dispersions seems to depend on the relative crystallization tendency of the pure substance. ${ }^{12}$ Moreover, the comparison of data on the mobility of the substance in the ASD with data on the mobility in the pure amorphous form will help to optimize the ASD design.

The present work is part of a research project developed in our laboratory on the study of the slow molecular mobility glass forming substances with pharmaceutical interest. ${ }^{13-16}$ This time the substances studied are valsartan, telmisartan and irbesartan, which are active non-peptide angiotensin II receptor antagonists used clinically for the treatment of cardiovascular diseases such as hypertension, cardiac insufficiency and cardiac arrhythmia. ${ }^{17-19}$

\footnotetext{
${ }^{a}$ CQFM - Centro de Química-Física Molecular and IN - Institute of Nanoscience and Nanotechnology, Instituto Superior Técnico, Universidade de Lisboa, 1049-001 Lisboa, Portugal. E-mail: mouraramos@tecnico.ulisboa.pt

${ }^{b} C Q E$ - Centro de Química Estrutural, Complexo I, Instituto Superior Técnico, Universidade de Lisboa, 1049-001 Lisboa, Portugal. E-mail: hdiogo@tecnico. ulisboa.pt
}

\section{(arrhythmia.17-19}


With differential scanning calorimetry (DSC) we will characterize the thermal behavior of the three studied substances: glass transition, melting, crystallization, glass forming ability, glass stability and tendency for crystallization on cooling from the equilibrium melt. We will also use DSC to characterize the structural relaxation of the three drugs. With TSDC we will analyze the slow molecular mobility in the amorphous solid form of the same substances.

\section{Experimental}

\subsection{Materials}

Valsartan, empirical formula $\mathrm{C}_{24} \mathrm{H}_{29} \mathrm{~N}_{5} \mathrm{O}_{3}$ (chemical structure on Fig. 1), CAS number 137862-53-4, molecular weight $M_{\mathrm{w}}=$ $435.52 \mathrm{~g} \mathrm{~mol}^{-1}$, was purchased from TCI, lot number VU4ZL (purity $>98.0 \%$ ), and was used without further purification. The DSC heat flow curve of the as received sample shows two signals occurring around $60{ }^{\circ} \mathrm{C}$ and $100{ }^{\circ} \mathrm{C}$. The first is clearly an endothermic overshoot overlapped with a change of heat capacity, which is the signature of an aged glass; the second has the appearance of an endothermic melting peak but its location is far from the reported melting temperature and the area is too small to match the enthalpy of fusion of the whole sample (see Table 1). Furthermore, the heat flow line between the two events reported at 60 and $100{ }^{\circ} \mathrm{C}$ is far to be flat, with a series of small overlapping signals, which may reveal an amorphous state with high level of structural arrangement (polyamorphism). In fact the results obtained from DSC, temperature modulated DSC and solid state NMR measurements showed that the fresh powder sample of valsartan is amorphous, which was confirmed by X-ray powder diffractometry. ${ }^{20-22}$ Furthermore, the analysis of different valsartan samples from different suppliers (from Poland, India and China) showed that the as received samples were

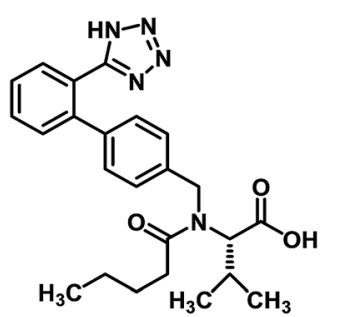

(a)

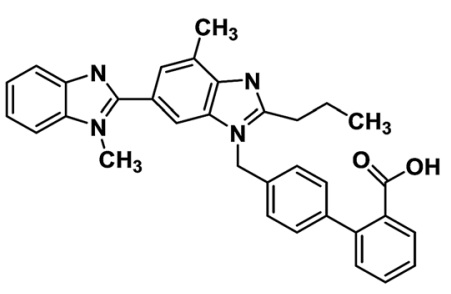

(b)

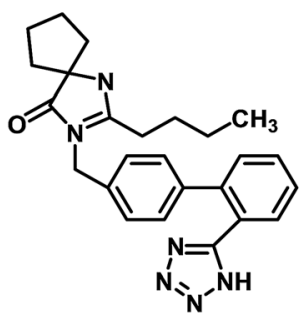

(c)

Fig. 1 The chemical structures of (a) valsartan, (b) telmisartan and (c) irbesartan. essentially identical. ${ }^{22}$ Valsartan crystals could only be prepared by controlling crystallization solvents and procedures, ${ }^{23}$ and the melting point and the melting enthalpy were found to be $T_{\text {fus }}=140{ }^{\circ} \mathrm{C}$ and $\Delta H_{\text {fus }}=43.6 \mathrm{~kJ} \mathrm{~mol}^{-1}$ (see Table 1).

Telmisartan, empirical formula $\mathrm{C}_{33} \mathrm{H}_{30} \mathrm{~N}_{4} \mathrm{O}_{2}$ (chemical structure on Fig. 1), CAS number: 144701-48-4, molecular weight: $514.62 \mathrm{~g} \mathrm{~mol}^{-1}$, was purchased from TCI, lot number $57 \mathrm{HKO}$ (purity $>98.0 \%$ ), and was used as received. The melting temperature of the as received crystalline powder, taken as the endothermic peak's maximum, was found to be $T_{\text {fus }}=271.0^{\circ} \mathrm{C}$ and the melting enthalpy was determined as $\Delta H_{\text {fus }}=54.2 \mathrm{~kJ}$ $\mathrm{mol}^{-1}$, in reasonable agreement with the published values (see Table 1).

Irbesartan, empirical formula: $\mathrm{C}_{25} \mathrm{H}_{28} \mathrm{~N}_{6} \mathrm{O}$ (chemical structure on Fig. 1), CAS number: 138402-11-6, molecular weight: $428.53 \mathrm{~g} \mathrm{~mol}^{-1}$, was purchased from TCI, lot number ZEMUM (purity $>98 \%$ ), and was used without further purification. The melting temperature of the as received crystalline powder was found to be $T_{\text {fus }}=183.0^{\circ} \mathrm{C}$ (maximum of the DSC melting peak at $10{ }^{\circ} \mathrm{C} \mathrm{min}{ }^{-1}$ ) and the melting enthalpy $\Delta H_{\text {fus }}=38.0 \mathrm{~kJ} \mathrm{~mol}^{-1}$, which corresponds to a particular crystalline polymorph, the socalled form A. The $45.9 \mathrm{~kJ} \mathrm{~mol}^{-1}$ value reported in the literature (see Table 1) seems to be too high. Indeed, different determinations carried out on samples from two different manufacturers (TCI and Sigma-Aldrich) led to $38 \mathrm{~kJ} \mathrm{~mol}^{-1}$, for this result has our full confidence. As discussed below, it is likely that the higher enthalpy of fusion reported before corresponds to a different polymorph.

2.1.1. Preparation of form $\mathbf{B}$ of irbesartan. The method of preparation was based on that described in the literature.$^{35} 0.8 \mathrm{~g}$ of the as received irbesartan (form A) was suspended into $10 \mathrm{~mL}$ of an aqueous solution of $\mathrm{HCl} 0.1 \mathrm{M}(\mathrm{pH}<2)$ and stirred during 48 hours at room temperature. Thereafter, the suspension was cooled to $c a .0{ }^{\circ} \mathrm{C}$, using an ice bath, and filtered through a porous glass plate. The resulting solid was first dried under nitrogen gas flux, and then inside a vacuum oven at $T=100{ }^{\circ} \mathrm{C}$ during 6 hours. The DSC thermogram showed that the melting temperature of this polymorph is $T_{\text {fus }}=186{ }^{\circ} \mathrm{C}$ (maximum of the DSC melting peak at $10{ }^{\circ} \mathrm{C} \mathrm{min}^{-1}$ ) and that the melting enthalpy is $\Delta H_{\text {fus }}=45.8 \mathrm{~kJ} \mathrm{~mol}^{-1}$, substantially higher than that of polymorph $\mathrm{A}$.

Fig. 2 shows the experimental powder X-ray diffraction patterns of the as received sample (top line, red color in the online edition, polymorph A) and of the synthetized sample (middle line, blue color in the online edition, polymorph B) together with the same pattern generated from the published $\mathrm{X}$ ray crystal structure of the polymorph B of irbesartan ${ }^{35}$ (bottom line, black in the online edition).

Comparing the bottom and middle lines show that the generated pattern of polymorph B fits very well that obtained experimentally; comparison of the top and middle lines highlights the different nature of the two crystalline forms of irbesartan. The authors who published the value $\Delta H_{\text {fus }}=45.9 \mathrm{~kJ}$ $\mathrm{mol}^{-1}$ (see Table 1) do not provide details on the synthesis of the irbesartan sample they received as a gift, so it is very likely to be polymorph $\mathrm{B}$. 
Table 1 Melting temperature (peak maximum), $T_{\text {fus, }}$ and enthalpy, $\Delta H_{\text {fus, }}$ of valsartan, telmisartan and irbesartan determined in the present work, compared with values reported in the literature

\begin{tabular}{lllr}
\hline & Valsartan & Telmisartan & Irbesartan (polymorph A) \\
\hline$T_{\text {fus }} /{ }^{\circ} \mathrm{C}$ & $140^{23}$ & $271^{a} ; 263 ;^{24} 264 ;^{25} 268 ;^{26,27} 269 ;^{28} 272^{29}$ & $183^{a} ; 184 ;^{30} 185^{31,32}$ \\
$\Delta H_{\text {fus }} / \mathrm{kJ} \mathrm{mol}^{-1}$ & $43.6^{23}$ & $54.2^{a} ; 52.7^{26}$ & $38.0^{a} ; 39.0 ;^{31} 45.9^{33,34}$ \\
${ }^{a}$ Present work. & & &
\end{tabular}

\subsection{Techniques}

2.2.1. Differential scanning calorimetry (DSC). The calorimetric measurements were performed with a 2920 MDSC system from TA Instruments Inc. The samples of $\sim 5-10 \mathrm{mg}$ were introduced in aluminium pans. The measuring cell was continuously purged with high purity helium gas at $30 \mathrm{~mL}$ $\min ^{-1}$. An empty aluminium pan, identical to that used for the sample, was used as the reference. Details of the calibration procedures are given elsewhere. ${ }^{36}$

2.2.2. Thermally stimulated depolarization currents (TSDC). Thermally stimulated depolarization current experiments were carried out with a TSC/RMA spectrometer (TherMold, Stamford, CT, USA) covering the range from $-170{ }^{\circ} \mathrm{C}$ to $+400{ }^{\circ} \mathrm{C}$. For TSDC measurements the sample (thickness of $\sim 0.5$ $\mathrm{mm}$ ) was placed between the disc-shaped electrodes $(7 \mathrm{~mm}$ diameter) of a parallel plane capacitor and immersed in an atmosphere of high purity helium (1.1 bar).

TSDC has a low equivalent frequency $\left(\sim 2 \times 10^{-3} \mathrm{~Hz}\right)$ so that it has a high resolution power and is sensitive to slow molecular motions (from $\sim 1$ to 500 seconds). Furthermore, the partial polarization (PP) experimental procedure (see below) allows probing narrow regions of the TSDC spectrum, i.e. narrowly distributed motional modes. The fact that the relaxation time of the motional processes is temperature dependent, and becomes longer as temperature decreases, allows to make it exceedingly long (freezing process) compared with the timescale of the experiment. As will be seen next this is the very core of the TSDC technique, which relies on the possibility of producing stable electrets at low temperatures by cooling down to those temperatures in the presence of a polarizing electric field; a clear and concise explanation of the experimental procedures provided by the technique of thermally stimulated currents is available ${ }^{37}$ and may be useful for the reader unfamiliar with this technique.

A brief explanation of the experimental protocol used in TSDC will however give the reader an easier understanding of the results presented and discussed in this paper. Two important parameters in a TSDC experiment are the polarization temperature, $T_{\mathrm{P}}$, at which the polarizing electric field is turned on, and the temperature $T_{\mathrm{P}}^{\prime}<T_{\mathrm{P}}$ at which the field is turned off (see Fig. 3). The difference $T_{\mathrm{P}}-T_{\mathrm{P}}^{\prime}$ is the width of the polarization window of the experiment.

If it is wide, the retained polarization (and of course the current peak that is the result of a TSDC experiment) will correspond to a complex set of energy distributed motional modes. Oppositely, the PP experiment where the polarizing field is applied in a narrow temperature interval, allows probing more narrowly distributed relaxation modes. In the conceptual limit of a very narrow polarization window, the experimental depolarization current peak is supposed to correspond to a single mode of relaxation. ${ }^{38}$ In the present work and in most of our previous ones we use polarization windows two degrees wide $\left(\Delta T=T_{\mathrm{p}}-T_{\mathrm{p}}^{\prime}=2{ }^{\circ} \mathrm{C}\right)$. The physical foundations of the TSDC experimental technique are presented in several publications $;^{\mathbf{3 9 - 4 1}}$ more recent review articles indicate a variety of research applications. ${ }^{42-46}$ The treatment of the raw

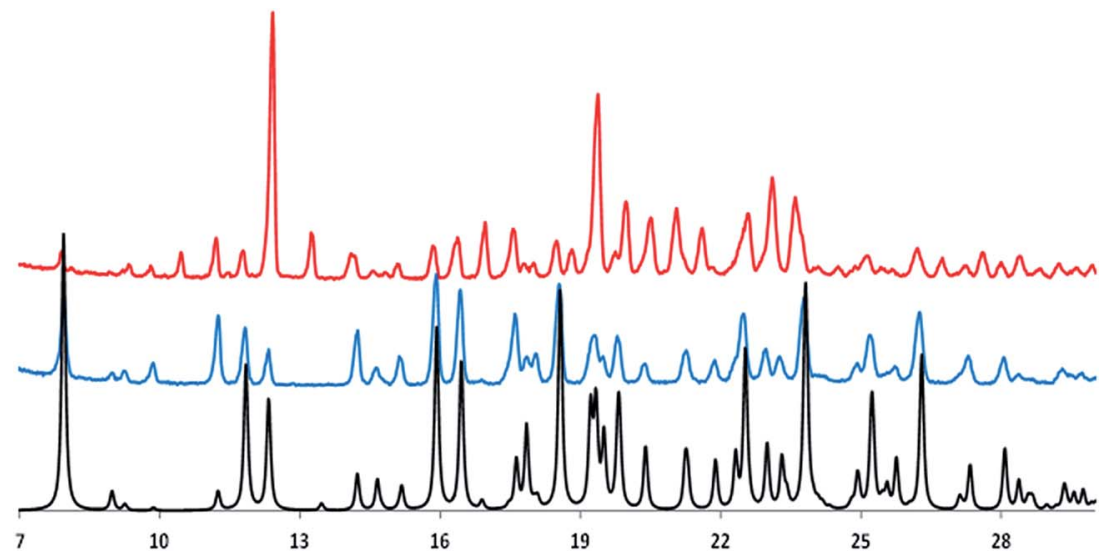

Fig. 2 X-ray diffraction patterns of the crystalline forms of irbesartan. Top line (red in the online edition): powder X-ray diffraction pattern of the as received sample, polymorph A. Middle line (blued in the online edition): powder X-ray diffraction pattern of the synthetized sample, polymorph B. Bottom line: powder X-ray diffraction pattern generated from the published X-ray crystal structure of the polymorph $B$. 


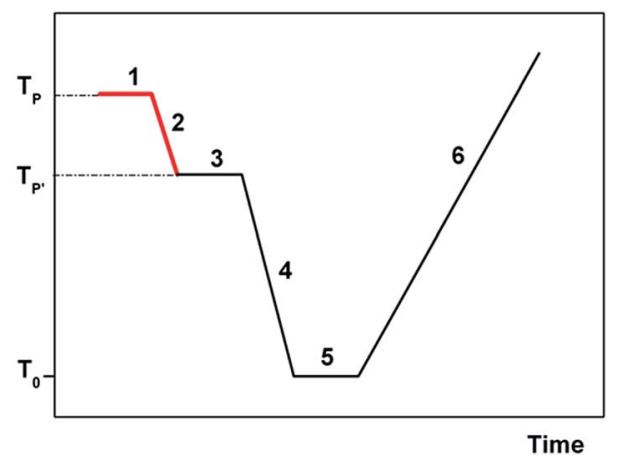

Fig. 3 Schematic diagram of the experimental procedure for a TSDC experiment. $\Delta T=T_{\mathrm{P}}-T_{\mathrm{P}}$ is the width of the polarizing window, typically between 0 and 5 degrees in a narrow window partial polarization (PP) experiment. The electric field is on in steps 1 and 2 (thicker lines, red in the online version) and the depolarization current is recorded during the constant rate heating process (step 6).

experimental results in order to determine the relaxation time and the kinetic parameters is explained succinctly in ref. 37.

Preparation of the amorphous solid samples for TSDC experiments. As stated above, valsartan is provided by the manufacturer in the form of an amorphous powder. For TSDC measurements a high compactness of the sample (high dipole density) is important to ensure a good signal to noise ratio. Therefore, the valsartan amorphous powder was placed on one of the metal electrodes, and the whole was heated to $130{ }^{\circ} \mathrm{C}$ (low viscosity metastable liquid), maintained at that temperature for 3 hours, and cooled down to room temperature inside an oven. The sample was thereby transformed into a transparent and colorless amorphous solid, and the sample/electrode set was then placed in the TSDC apparatus in contact with the second electrode and under helium atmosphere.

The samples of telmisartan and irbesartan in the amorphous solid state were prepared as follows. A given amount of the as received crystalline sample is placed on one of the electrodes, the whole placed in a vacuum oven, submitted to reduced pressure and heated to $\sim\left(T_{\text {fus }}+10\right)$. After ten minutes isotherm, the temperature of the oven has been lowered to room temperature, put back at normal pressure, transferred to the TSDC sample chamber in contact with the second electrode, and placed under helium atmosphere. Note that, since the natural cooling of the oven under vacuum is necessarily very slow, this procedure wherein the amorphous sample is prepared outside the TSDC chamber is only achievable if the test substance exhibits a high glass forming ability (high resistance to crystallization on cooling from the melt), which is the case for telmisartan and irbesartan.

2.2.3. Powder X-ray diffractometry. Powder X-ray diffraction (PXRD) patterns of samples were recorded at $297 \pm 1 \mathrm{~K}$, on a D8 Advance Bruker X-ray diffractometer (Karlsruhe, Germany) operating in the $\theta-2 \theta$ mode. The radiation originated by the source $(40 \mathrm{kV}$ and $40 \mathrm{~mA})$ goes through a Ni-filtered $\mathrm{Cu}-\mathrm{K} \alpha(\lambda=$ $1.5406 \AA$ ) and a LinxEye detector. Diffraction data were collected in the $2 \theta$ range from $7^{\circ}$ to $35^{\circ}$, in $0.02^{\circ}$ steps, and with an overall scan time of approximately $15 \mathrm{~min}$. The samples were mounted on a glass sample holder. The program MERCURY 3.026 (ref. 47) was used to simulate diffraction patterns from published single crystal X-ray diffraction data.

\section{Results and discussion}

\subsection{Particular aspects of the thermal behavior of the studied substances}

3.1.1. Valsartan. Subjecting the amorphous powder valsartan supplied by the manufacturer to a DSC heating ramp from $-80{ }^{\circ} \mathrm{C}$ up to $150{ }^{\circ} \mathrm{C}$, the complex heat flow line between 60 and $100{ }^{\circ} \mathrm{C}$ described before in Section 2.1 is observed, and no melting traces are observed in the vicinity of $T_{\text {fus }}=140{ }^{\circ} \mathrm{C}$. On the second heating run the heat flow line is simplified, it becomes flat in the temperature interval between 60 and $100{ }^{\circ} \mathrm{C}$, with only one event observed: a glass transition at $69{ }^{\circ} \mathrm{C}$ with $\Delta C_{\mathrm{p}}=0.42 \pm 0.01 \mathrm{~J} \mathrm{~K}^{-1} \mathrm{~g}^{-1}$ (see Table 2). Comparing the glass transition signals obtained in the first two runs shows that it moves from approximately $60{ }^{\circ} \mathrm{C}$ to $69^{\circ} \mathrm{C}$ and that the endothermic structural relaxation overshoot superimposed on the heat flow step decreases sharply in intensity. This indicates that the as received sample was heavily aged and plasticized due to moisture. In all subsequent heating/cooling cycles carried out with the valsartan sample only the step signal of the glass transition is observed, with no trace of crystallization during both cooling and heating (for heating/cooling rates from 1 to $20{ }^{\circ} \mathrm{C} \min ^{-1}$ ). The fact that valsartan does not crystallize on cooling from the melt indicates that it has a very high glass forming ability. It has also high glass stability since it does not show any tendency to crystallize on heating from the glass or from the metastable liquid state. As stated above, valsartan crystals could only be prepared by recrystallization from solvents under a careful control of the crystallization solvents and procedures. ${ }^{23}$

Table 2 The features of the glass transition of valsartan, telmisartan and irbesartan

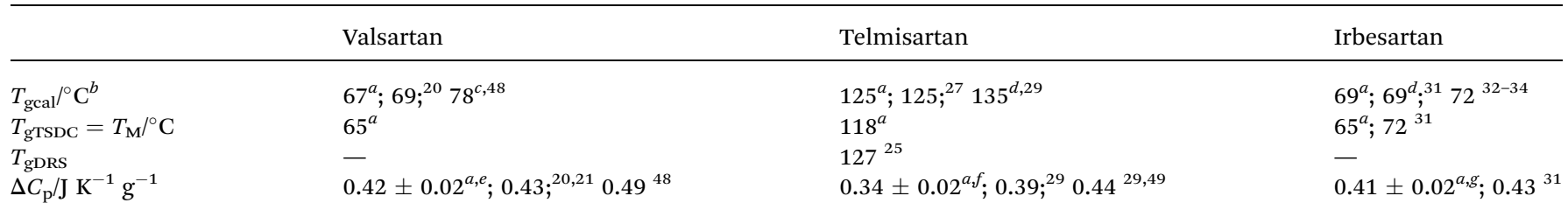

${ }^{a}$ Determined in the present work. ${ }^{b}$ Extrapolated onset temperature at $10^{\circ} \mathrm{C} \min ^{-1}$, except otherwise stated. ${ }^{c}$ Obtained by MTDSC. ${ }^{d}$ Midpoint of the step signal. ${ }^{e}$ Mean over 122 determinations. ${ }^{f}$ Mean over 17 determinations. ${ }^{g}$ Mean over 59 determinations. 
3.1.2. Telmisartan. Two polymorphic forms of telmisartan have been detected, ${ }^{28}$ with melting temperatures $183{ }^{\circ} \mathrm{C}$ and $269{ }^{\circ} \mathrm{C}$. The melting temperature of our as received sample (Table 1) shows that it corresponds to the most stable one (higher $T_{\text {fus }}$ ), the so-called form A. On the other hand, the glass transition was determined as $T_{\mathrm{g}}=125{ }^{\circ} \mathrm{C}$ with $\Delta C_{\mathrm{p}}=0.34 \pm$ $0.02 \mathrm{~J} \mathrm{~K}^{-1} \mathrm{~g}^{-1}$ in reasonable agreement with the values reported in the literature (Table 2). Telmisartan revealed no tendency to crystallize upon cooling from the melt. We have found, however, according to other authors, ${ }^{29}$ the existence of a relatively slow cold crystallization with onset at about $T_{\mathrm{cc}}=200{ }^{\circ} \mathrm{C}$ and maximum speed at $\sim 220^{\circ} \mathrm{C}$ (at a heating rate $10^{\circ} \mathrm{C} \mathrm{min}{ }^{-1}$ ). The fact that $25^{\circ} \mathrm{C} \ll T_{\mathrm{g}} \ll T_{\text {cc }}$ ensures that glassy telmisartan is very stable under the normal ambient conditions.

3.1.3. Irbesartan. An US Patent ${ }^{50}$ disclosed that irbesartan can exist in two polymorphic crystalline forms, named A and B. As noted before, the melting thermodynamic properties obtained for the as received sample and shown in Table 1 correspond to form A. The crystalline form B is reported to have $T_{\text {fusB }}$ $=189^{\circ} \mathrm{C}$ and $\Delta H_{\text {fusB }}=49.7 \mathrm{~kJ} \mathrm{~mol}^{-1},{ }^{31}$ values that compare with those of the sample synthetized in our laboratory and referred before: $T_{\text {fusB }}=186{ }^{\circ} \mathrm{C}$ and $\Delta H_{\text {fusB }}=45.8 \mathrm{~kJ} \mathrm{~mol}^{-1}$.

A pair of polymorphs necessarily belongs to one of two categories, called monotropic or enantiotropic. ${ }^{51,52}$ Irbesartan is supposed to have a monotropic pair of polymorphs. ${ }^{31}$ In a monotropic system no endothermic solid-solid transition is observed from the lower melting temperature form to the higher melting one. In fact, the DSC thermogram of irbesartan does not show any evidence of this transition below the melting temperature of both polymorphs. This behavior manifests itself in other ways, also observed in irbesartan, namely that the polymorph with higher melting temperature also has higher enthalpy and entropy of fusion. Comparing these thermodynamic properties for the polymorphs A and B suggests that polymorph B is thermodynamically more stable (higher melting temperature and enthalpy), while polymorph A (sample received from the manufacturer) is metastable. This means that the reaction mechanism of the chemical synthesis of irbesartan favors the formation of the metastable form $\mathrm{A}$, and that, under the storage conditions (room temperature, normal pressure), there are no efficient paths to transform A into the most stable $B$. We found however that the transformation of $A$ into $B$ is possible through a mechanism that implies the melting of $\mathrm{A}$ followed by the cold crystallization of B. This is shown in Fig. 4 where four conventional DSC thermograms taken at different heating rates are presented.

In the heat flow line (a), at $10{ }^{\circ} \mathrm{C} \mathrm{min}^{-1}$, only the melting peak of $\mathrm{A}$ is visible, whereas in lines (c) and (d), at 1 and $0.2^{\circ} \mathrm{C}$ $\min ^{-1}$ respectively, the melting peaks of polymorphs $\mathrm{A}$ and $\mathrm{B}$ are clearly seen. Line (b) at $2.5^{\circ} \mathrm{C} \mathrm{min}^{-1}$, on the other hand, only shows the melting of a small amount of B. Note that the position of the peaks is moved to higher temperatures with increasing heating rate, as it should be. The conclusion drawn from the results shown in Fig. 4 is that polymorph B forms in appreciable amounts only at very slow ramps, which suggests that the cold crystallization of irbesartan has slow kinetics.

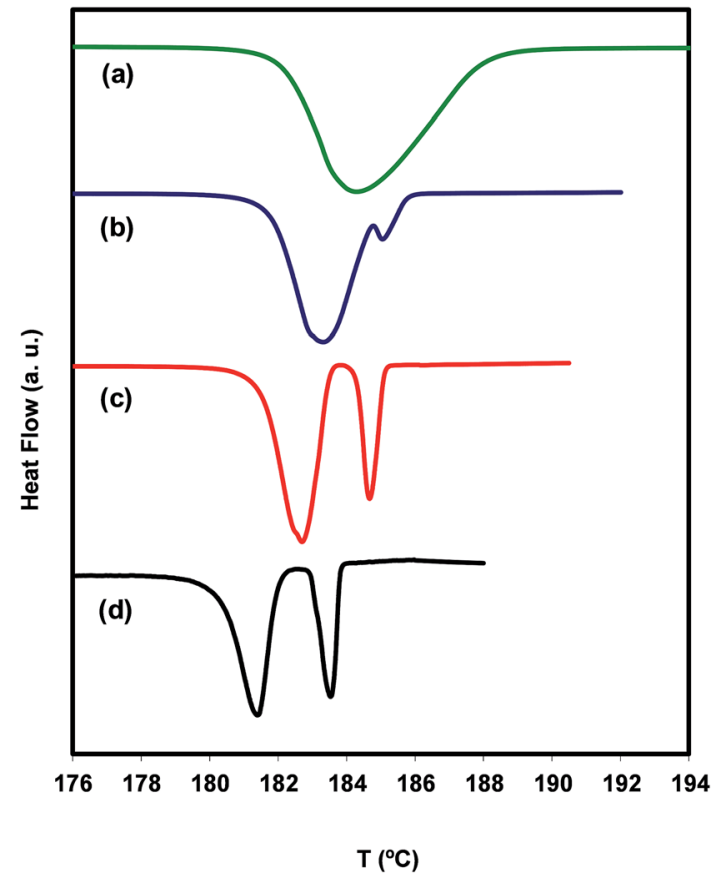

Fig. 4 Conventional DSC thermograms of irbesartan starting from below the melting temperature of polymorph $\mathrm{A}$ and taken at different heating rates: (a) $10^{\circ} \mathrm{C} \mathrm{min}^{-1}$ (green in the online edition); (b) $2.5^{\circ} \mathrm{C}$ $\mathrm{min}^{-1}$ (blue in the online edition); (c) $1{ }^{\circ} \mathrm{C} \mathrm{min}^{-1}$ (red in the online edition); (d) $0.2^{\circ} \mathrm{C} \mathrm{min}^{-1}$ (black in the online edition).

Under these conditions, it is natural for the exothermic effect of the cold crystallization to be compensated for by the endothermic effect of the simultaneous melting of A, leading to the concealment of the cold crystallization signal in the DSC thermogram.

A more detailed view of what is happening during a very slow heating of the polymorph A is provided by the result of a DSC experiment carried out in the step-by-step mode (see Fig. 5).

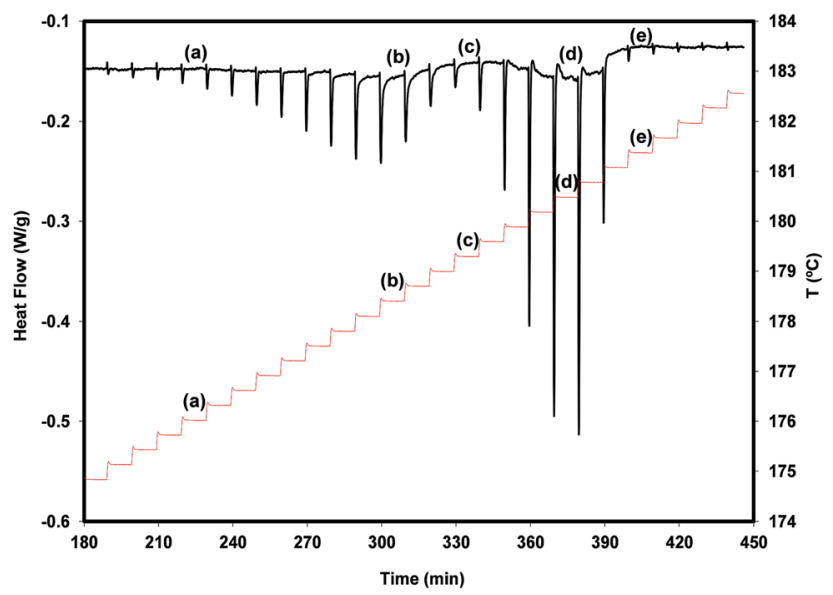

Fig. 5 Step by step DSC experiment on the melting temperature region of irbesartan. The step line (red in the online edition) represents the experimental protocol, constituted by isotherms of 10 minutes separated by temperature jumps of $0.3^{\circ} \mathrm{C}$. The other curve is the experimental result (heat flow as a function of time and of temperature). 


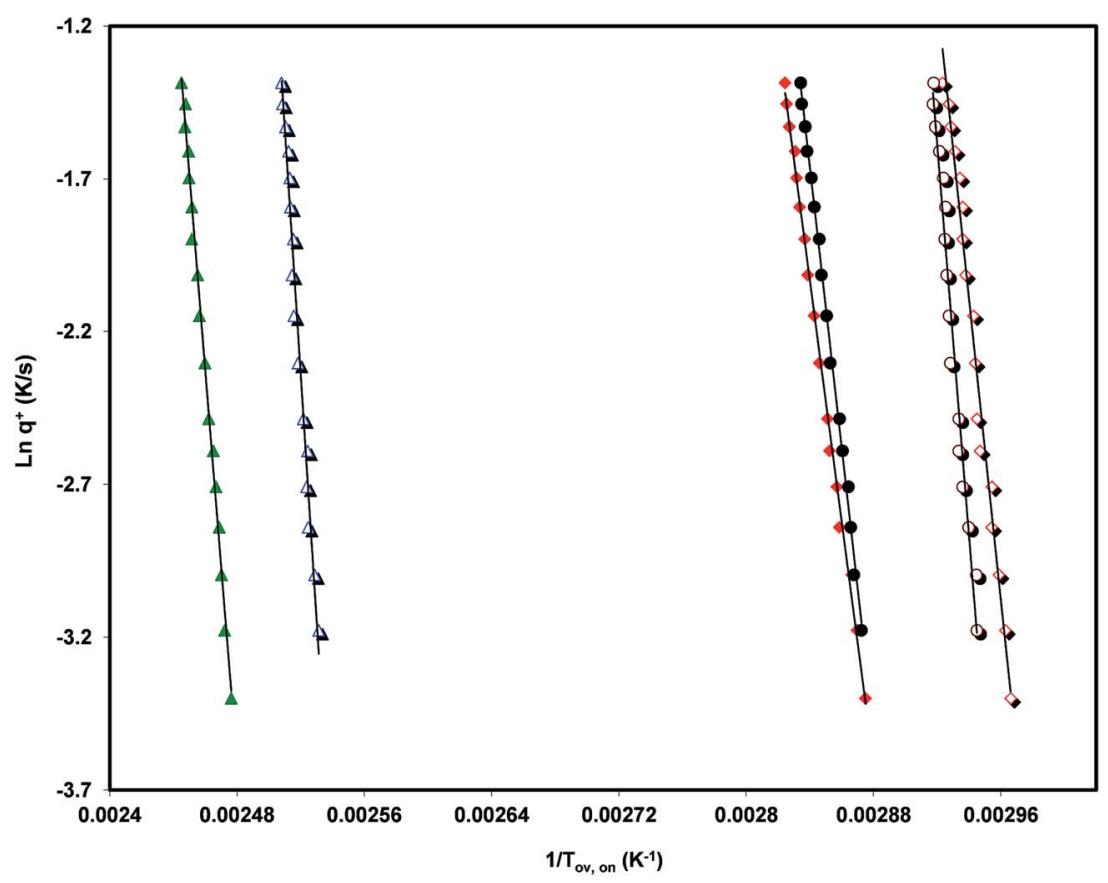

Fig. 6 "Arrhenius plots" of the logarithm of the heating rate, $q^{+}$, as a function of $1000 / T_{\text {ov }}$ (full symbols) and of $1000 / T_{\text {on }}$ (empty symbols). The triangles refer to telmisartan (green in the online edition), the diamonds to valsartan (red in the online edition), and the circles to irbesartan. The experiments were designed in such a way that the ratio between the heating rate, $q^{+}$, and the previous cooling rate, $q^{-}$, was unity: $q^{-} / q^{+}=1$.

We find from this figure that the melting of A begins at $175.7^{\circ} \mathrm{C}$ (step a), shows maximum rate at $178.4^{\circ} \mathrm{C}$ (step b) and finishes at about $179.3^{\circ} \mathrm{C}$ (step c). The melting of B, crystallized from the melt of A during the melting process of this polymorph, occurs between step c and step e $\left(181.4{ }^{\circ} \mathrm{C}\right)$ and shows maximum rate at $180.5^{\circ} \mathrm{C}$ (step d). The melting processes of the two polymorphs appear as well separated, and no signal clearly attributable to the cold crystallization of B is observed. These results suggest that, in addition to being chemically feasible (process described above), the $\mathrm{A} \rightarrow \mathrm{B}$ transformation can also be carried out by thermal treatments.

Irbesartan showed, as valsartan, very high glass forming ability and glass stability. The glass transition of irbesartan was determined as $T_{\mathrm{g}}=69^{\circ} \mathrm{C}$ with $\Delta C_{\mathrm{p}}=0.41 \pm 0.02 \mathrm{~J} \mathrm{~K}^{-1} \mathrm{~g}^{-1}$ in reasonable agreement with the values reported in the literature (Table 2).

\subsection{DSC characterization of the dynamics of the glass transition}

Analyzing the influence of the heating rate on the temperature location of the DSC glass transition signature allows the characterization of the kinetics of the glass transition relaxation. The basic equation to do this is: ${ }^{53}$

$$
\frac{\mathrm{d}(\ln q)}{\mathrm{d}\left(1 / T_{\mathrm{x}}\right)}=-\frac{E_{\mathrm{a}}\left(T_{\mathrm{g}}\right)}{R}
$$

where $R$ is the gas constant, $T_{\mathrm{x}}$ is temperature that defines the position of the DSC glass transition signal, and $E_{\mathrm{a}}\left(T_{\mathrm{g}}\right)$ the activation energy of the structural relaxation. The DSC signal of the glass transition on heating displays different specific points ${ }^{54}$ that can be used to define its temperature location, $T_{\mathrm{x}}$. We will consider two of them whose temperatures can be defined more precisely. The first, more conventional, is the extrapolated onset temperature (temperature at the intersection of the extrapolated baseline and the tangent taken at the point of maximum slope), $T_{\text {on }}{ }^{54}$ The second, recently considered by Svodoba, ${ }^{55,56}$ is the temperature of the minimum of the heat flow overshoot (in the "exo up" configuration) in the glass transition signal, $T_{\mathrm{ov}}$. To determine $E_{\mathrm{a}}\left(T_{\mathrm{g}}\right)$ using eqn (1) we need to obtain $T_{\mathrm{on}}$ and $T_{\mathrm{ov}}$ for different heating rates, $q^{+}$, and this is done in cooling/heating cycles such that each heating scan is immediately preceded by a cooling scan at the same rate $\left(q^{-} / q^{+}\right.$is unity).$^{53,57}$ The results are plotted on Fig. 6 . The heating rate dependences of $T_{\mathrm{ov}}$ and $T_{\text {on }}$ are shown respectively as full and open symbols; the points relative to telmisartan are shown as triangles (green in the online edition, determination coefficients $r^{2}=0.994$ and $r^{2}=$ 0.977 respectively), those relative to irbesartan are diamonds (red in the online edition, determination coefficients $r^{2}=0.997$ and $r^{2}=0.972$ respectively), and those of valsartan are circles (determination coefficients $r^{2}=0.996$ and $r^{2}=0.984$ ).

The values of the activation energy of the structural relaxation calculated using eqn (1) for $T_{\mathrm{x}}=T_{\mathrm{ov}}$ and for $T_{\mathrm{x}}=T_{\mathrm{on}}$ are shown in Table 3, and the dynamic fragilities, defined as ${ }^{58,59}$

$$
m=\frac{E_{\mathrm{a}}\left(T_{\mathrm{g}}\right)}{(\ln 10) \times R T_{\mathrm{g}}},
$$

were calculated from those values.

From the comparison of the values present in Table 3 we can see the tendency, already observed, ${ }^{15,16}$ for $T_{\text {on }}$ to lead to values of $m_{\mathrm{DSC}}$ higher than those obtained from $T_{\mathrm{ov}}$. We can also 
Table 3 Thermodynamic and dynamic fragilities of valsartan, telmisartan and irbesartan

\begin{tabular}{llll}
\hline & Valsartan & Telmisartan & Irbesartan \\
\hline$E_{\mathrm{a}}\left(T_{\mathrm{g}}\right)_{\mathrm{DSC}} / \mathrm{kJ} \mathrm{mol}^{-1}$ & $328^{a, b} ; 409^{a, c}$ & $533^{a, b} ; 667^{a, c}$ & $381^{a, b} ; 533^{a, c}$ \\
$m_{\mathrm{DSC}}$ & $50^{a, b} ; 63^{a, c}$ & $70^{a, b} ; 88^{a, c}$ & $58^{a, b} ; 82^{a, c}$ \\
$m_{\mathrm{TSDC}}$ & $47^{a}$ & $62^{a}$ & $48^{a}$ \\
$m_{\mathrm{DRS}}$ & - & $87^{25}$ & -
\end{tabular}

${ }^{a}$ Determined in the present work. ${ }^{b}$ Estimated from the heating rate dependence of the overshoot peak temperature location, $T_{\mathrm{ov}}$. ${ }^{c}$ Estimated from the heating rate dependence of the extrapolated onset temperature, $T_{\mathrm{on}}$.

observe that the three drugs studied are relatively strong liquids, and that telmisartan is among them the one with a markedly superior fragility.

\subsection{The molecular mobility studied by TSDC}

In order to study the molecular mobility by TSDC, it is first necessary to detect and localize the different relaxations in the temperature axis, which is done by scanning the temperature domain (from the lower limit of the apparatus, often $\sim-150{ }^{\circ} \mathrm{C}$, up to some degrees above $T_{\mathrm{g}}$ ) using wide polarization window experiments. Series of partial polarization (PP) experiments are then carried out, which lead to the analysis of the distribution of relaxation times of the different mobilities detected in the material under study.

3.3.1. The glass transition relaxation. Fig. 7 shows a series of PP current peaks in the temperature region of the glass to metastable liquid transformation, obtained for telmisartan. The peak in this series with a higher intensity is the subject of particular attention because it is the one where a higher extent of polarization was allowed to be "frozen-in", and it corresponds to a situation in which the electric field was allowed to polarize nearly all the higher activation energy motional modes associated with the glass transition relaxation. The temperature of maximum intensity, or temperature location of this singular peak, which is shown in Fig. 7(b), will be represented by $T_{\mathrm{M}}$, to distinguish from the temperature location of the other peaks generically represented by $T_{\mathrm{m}}$. $T_{\mathrm{M}}$ represents the lower limit (the onset) of the transition range between the non-equilibrium glass and the phase into which it transforms by heating, and hence is considered is considered as the glass transition temperature provided by the TSDC technique at the heating rate of the experiment $\left(T_{\mathrm{M}}=T_{\mathrm{g}}\right)$.

For the peak in Fig. $7(\mathrm{~b})$ we have $T_{\mathrm{M}}=T_{\mathrm{gTSDC}}=117.7^{\circ} \mathrm{C}$. The temperature dependent relaxation time, $\tau(T)$, associated to a given PP peak (i.e. to a given narrowly distributed motional mode) is obtained by a standard treatment briefly explained in Section 2 of ref. 37. Fig. 7 (b) shows the $\log _{10} \tau(T)$ versus $1 / T$ line associated to the PP peak located at $T_{\mathrm{M}}$; it does not deviate much from linearity but this does not always happen, especially in the case of very fragile liquids in which it has a certain curvature. The adjustment of this line to a suitable equation (Arrhenius, Vogel-Fulcher-Tammann, Williams-Landel-Ferry, ...) allows to obtain the kinetic parameters that characterize the
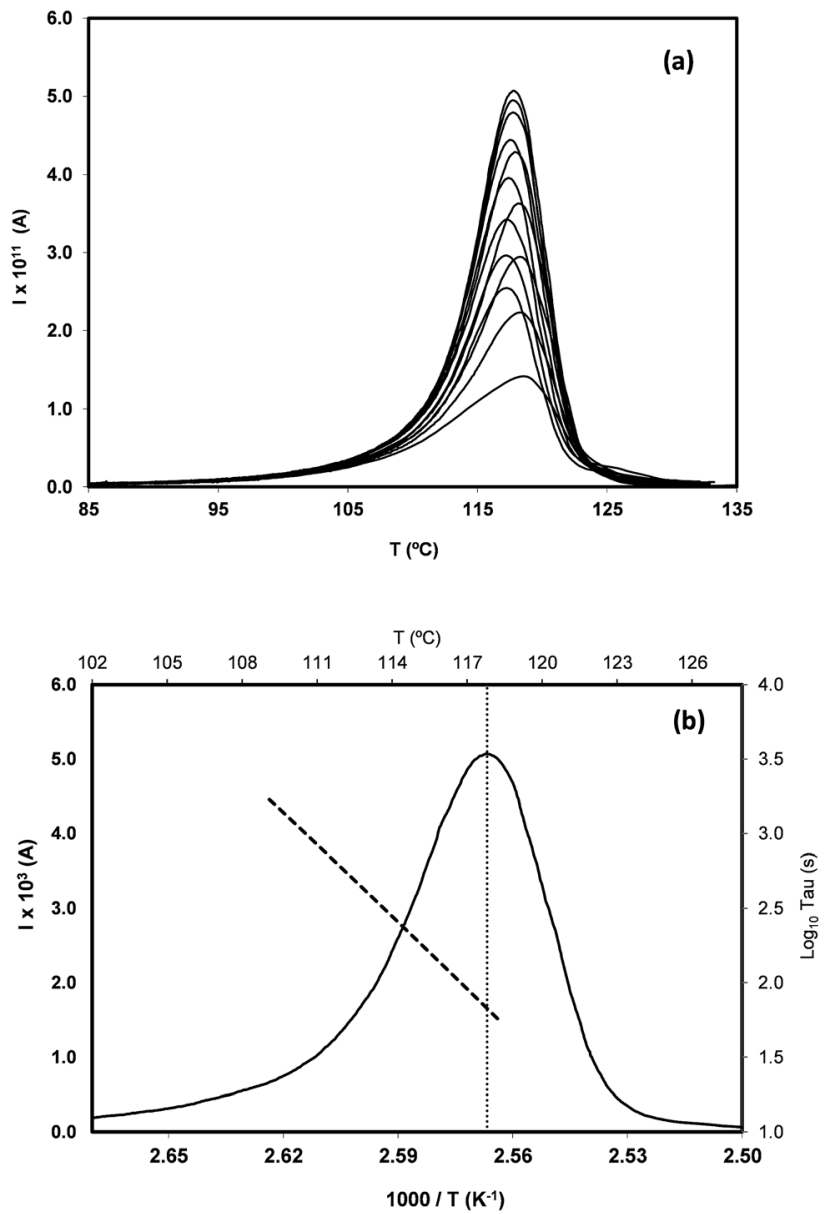

Fig. 7 (a) Partial polarization (PP) peaks of the glass transition relaxation of telmisartan, obtained with polarization temperatures, $T_{p}$, from $111^{\circ} \mathrm{C}$ to $124^{\circ} \mathrm{C}$. In all the experiments the width of the polarization window was $\Delta T=2{ }^{\circ} \mathrm{C}$, the polarizing electric field strength was $E=$ $250 \mathrm{~V} \mathrm{~mm}^{-1}$, and the heating rate was $q=4^{\circ} \mathrm{C} \mathrm{min}^{-1}$. (b) Peak with maximum intensity in (a), $T_{p}=118{ }^{\circ} \mathrm{C}$, located at $T_{M}=117.7^{\circ} \mathrm{C}$ (temperature of the dotted line). The dashed line is the corresponding $\log _{10} \tau(T)$ versus $1 / T$ line. The current intensity scale is on the right, the relaxation time scale is on the left.

motional mode. The activation energy at the maximum temperature, $T_{\mathrm{m}}$, of the corresponding PP peak, $E_{\mathrm{a}}\left(T_{\mathrm{m}}\right)$, is commonly used to kinetically characterize each partial polarization mode:

$$
E_{\mathrm{a}}\left(T_{\mathrm{m}}\right)=\ln 10 \times R \times\left(\frac{\partial \log _{10} \tau(T)}{\partial \frac{1}{T}}\right)_{T=T_{\mathrm{m}}}
$$

For the PP motional mode considered in Fig. 7(b) we have $E_{\mathrm{a}}\left(T_{\mathrm{M}}\right)=E_{\mathrm{a}}\left(T_{\mathrm{g}}\right)=462 \mathrm{~kJ} \mathrm{~mol}^{-1}$, so the dynamic fragility of telmisartan calculated from eqn (2) is $m=62$. Using a similar procedure we obtain $m_{\mathrm{TSDC}}=47$ and $m_{\mathrm{TSDC}}=48$ for valsartan and irbesartan (see Table 3). Despite the relatively pronounced dispersion of the $m$ values presented in Table 3, telmisartan is, among the three vitrifiable liquids studied, the one with the highest fragility index. 
Fig. 8 is a relaxation map that displays the activation energy, $E_{\mathrm{a}}\left(T_{\mathrm{m}}\right)$, of the PP motional modes as a function of their temperature location, $T_{\mathrm{m}}$, for the different relaxations detected in the amorphous solid state of the studied drugs. The dotted line is the so-called zero activation entropy line that depicts the behavior of the local and non-cooperative relaxations (see Section 3 of ref. 37). In this figure the points in the vicinity of the zero entropy line correspond to the local movements of the secondary relaxations, whereas those that move away from it correspond to the cooperative movements of the glass transition. For a given substance, the amplitude of the deviation to the zero entropy line is proportional to its fragility index, while the temperature of the points with the greatest distance from that line is a reasonable estimate of the glass transition temperature.

3.3.2. The secondary relaxations. The points in Fig. 8 that correspond to the local secondary mobility are located, as stated above, in the vicinity of the zero entropy line. Fig. 8 thus suggests the existence of an extensive secondary relaxation in valsartan (circles in the temperature range from -30 to $+60{ }^{\circ} \mathrm{C}$ ). The secondary relaxation of valsartan, of which some PP modes are shown in Fig. 9, has energies distributed between 65 and 90 $\mathrm{kJ} \mathrm{mol}^{-1}$. In irbesartan, on the other hand, only an incipient secondary mobility with extremely low intensity was detected in a very narrow temperature range (at $\sim 8{ }^{\circ} \mathrm{C}$, see Fig. 8 ), which is in line with a study of irbesartan by TSDC previously published in the literature. ${ }^{31}$

Although no secondary mobility has been detected by TSDC in telmisartan, a study by dielectric relaxation spectroscopy $(\mathrm{DRS})^{25}$ reveals its existence, and shows that it obeys the Arrhenius equation, $\tau_{\beta}=\tau_{\infty} \exp \left(E_{\beta} / R T\right)$, with $\log _{10} \tau_{\infty}=-15.67$ and $E_{\beta}=81.8 \mathrm{~kJ} \mathrm{~mol}^{-1}$. Based on these results, and by simple manipulation of the Arrhenius equation, it can be seen that the

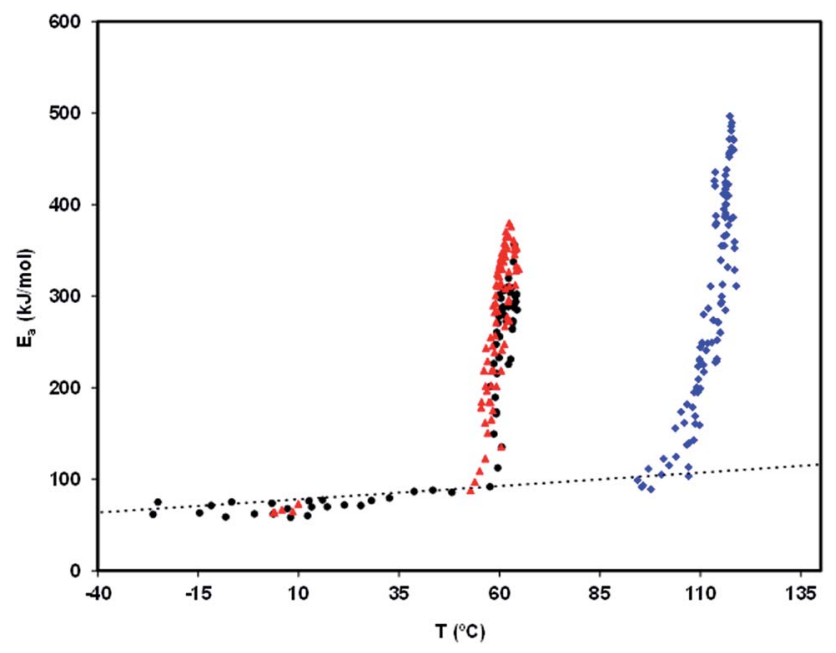

Fig. 8 TSDC relaxation map of valsartan, telmisartan and irbesartan: activation energy, $E_{a}\left(T_{m}\right)$, of the PP components of the mobility as a function of the temperature location, $T_{m}$, of the corresponding current peak. The circles correspond to valsartan, the triangles (red in the online edition) to irbesartan, and the diamonds (blue in the online edition) to telmisartan. The dotted line is the zero entropy line.

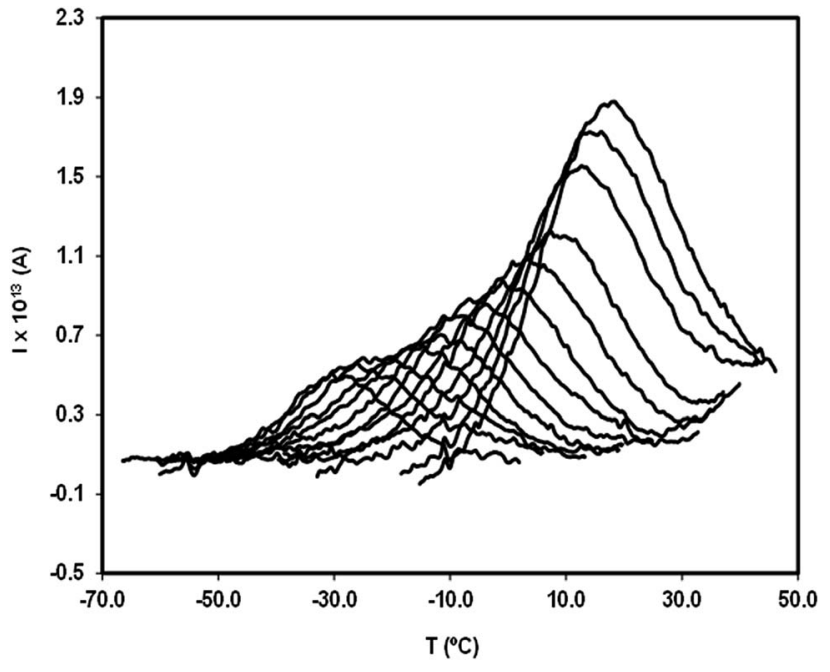

Fig. 9 Partial polarization (PP) peaks of the secondary relaxation of valsartan, obtained with polarization temperatures, $T_{\mathrm{p}}$, from $-31^{\circ} \mathrm{C}$ to $+13{ }^{\circ} \mathrm{C}$. The width of the polarization window was $\Delta T=2{ }^{\circ} \mathrm{C}$, the polarizing electric field strength was $E=450 \mathrm{~V} \mathrm{~mm}^{-1}$, and the heating rate was $q=4^{\circ} \mathrm{C} \min ^{-1}$.

relaxation time $\tau_{\beta}$ should have values between 10 and 500 seconds (time range in the time window of the TSDC technique) in the temperature interval between -15 and $-40{ }^{\circ} \mathrm{C}$. The fact that TSDC proves incapable of detecting any mobility in telmisartan in this temperature range probably arises from the low strength of the rotating dipoles and/or from the weak amplitude of the possible reorientational motions.

In this context, the technique of dielectric spectroscopy appears as a more sensitive tool to detect subtle reorientational movements.

\section{Conclusions}

The thermal behavior of valsartan, telmisartan and irbesartan, particularly their crystallization tendency on heating from the amorphous solid as well as on cooling from the equilibrium liquid, was investigated by differential scanning calorimetry. It was found that valsartan and irbesartan have very high glass forming ability and glass stability. Telmisartan revealed no tendency to crystallize upon cooling from the melt but it shows a relatively slow cold crystallization with onset at $200{ }^{\circ} \mathrm{C}$, so that it has high glass forming ability and moderate glass stability.

Irbesartan has two monotropic polymorphic forms with close melting temperatures and with significantly different enthalpies of fusion. It is known that polymorph A (commercially available, metastable) can be transformed into $\mathrm{B}$ chemically; we have further shown that the $\mathrm{A} \rightarrow \mathrm{B}$ transformation can occur through cold crystallization of $\mathrm{B}$ from the melt of A, in a very slow heating process.

The slow molecular mobilities in amorphous valsartan, telmisartan and irbesartan were studied by differential scanning calorimetry and thermally stimulated depolarization currents, allowing the determination of the thermodynamic and kinetic parameters of the different relaxations. 
The analysis of the heating rate effect on the temperature location of the DSC glass transition step allowed the determination of the activation energy of the structural relaxation and of the steepness or fragility index of the studied amorphous drugs.

The distributions of relaxation times of the different mobilities detected in the studied amorphous solid substances were characterized by TSDC for the secondary and main relaxations. Fast secondary motions were detected in valsartan but not in telmisartan and irbesartan. The analysis of the $\alpha$-relaxation provided the values of the dynamic fragility, $m_{\mathrm{TSDC}}$; the three substances behave as moderately strong glass-formers, telmisartan being the one with the highest dynamic fragility.

\section{Acknowledgements}

We thank Dr Maria de Fátima Piedade for performing the powder X-ray analysis of the irbesartan polymorphs. This work was partially supported by Fundação para a Ciência e a Tecnologia (FCT), Portugal (Projects UID/NAN/50024/2013 and UID/ QUI/00100/2013).

\section{References}

1 B. C. Hancock, J. Pharm. Pharmacol., 2002, 54, 737-746.

2 S. B. Murdande, M. J. Pikal, R. M. Shanker and R. H. Bogner, J. Pharm. Sci., 2010, 99, 1254-1264.

3 S. B. Murdande, M. J. Pikal, R. M. Shanker and R. H. Bogner, Pharm. Res., 2010, 27, 2704-2714.

4 G. P. Johari and R. M. Shanker, Thermochim. Acta, 2014, 598, 16-27.

5 K. Kothari, V. Ragoonanan and R. Suryanarayanan, Mol. Pharm., 2014, 11, 3048-3055.

6 S. P. Bhardwaj, K. K. Arora, E. Kwong, A. Templeton, S. D. Clas and R. Suryanarayanan, Mol. Pharm., 2013, 10, 694-700.

7 S. Bhattacharya and R. Suryanarayanan, J. Pharm. Sci., 2009, 98, 2935-2953.

8 F. Paladi and M. Oguni, J. Phys.: Condens. Matter, 2003, 15, 3909-3917.

9 Y. Xu, J. F. Carpenter, M. T. Cicerone and T. W. Randolph, Soft Matter, 2013, 9, 7855-7865.

10 A. Singh, Z. A. Worku and G. Van den Mooter, Expert Opin. Drug Delivery, 2011, 8, 1361-1378.

11 Y. He and C. Ho, J. Pharm. Sci., 2015, 104, 3237-3258.

12 P. J. Marsac, H. Konno and S. Taylor, Pharm. Res., 2006, 23, 2306-2316.

13 N. T. Correia, J. J. Moura Ramos, M. Descamps and G. Collins, Pharm. Res., 2001, 18, 1767-1774.

14 H. P. Diogo and J. J. Moura Ramos, J. Non-Cryst. Solids, 2015, 429, 202-207.

15 M. T. Viciosa, J. J. Moura Ramos and H. P. Diogo, J. Pharm. Sci., 2016, 105, 3573-3584.

16 J. J. Moura Ramos and H. P. Diogo, Int. J. Pharm., 2016, 501, 39-48.

17 R. Dina and M. Jafari, Am. J. Health-Syst. Pharm., 2000, 57, 1231-1241.
18 M. Volpe, Expert Rev. Cardiovasc. Ther., 2012, 10, 1061-1072. 19 H. G. Pouleur, Am. J. Hypertens., 1997, 10, 318S-324S.

20 M. Skotnicki, A. Gawel, P. Cebe and M. Pyda, Drug Dev. Ind. Pharm., 2013, 39, 1508-1514.

21 M. Skotnicki, J. A. Aguilar, M. Pyda and P. Hodgkinson, Pharm. Res., 2015, 32, 414-429.

22 M. Skotnicki, D. C. Apperley, J. A. Aguilar, B. Milanowski, M. Pyda and P. Hodgkinson, Mol. Pharm., 2016, 13, 211222.

23 J. R. Wang, X. Wang, L. Lu and X. Mei, Cryst. Growth Des., 2013, 13, 3261-3269.

24 R. Saber, A. Attia and W. Salem, Adv. Pharm. Bull., 2014, 4, 283-287.

25 K. Adrjanowicz, Z. Wojnarowska, P. Wlodarczyk, K. Kaminski, M. Paluch and J. Mazgalski, Eur. J. Pharm. Sci., 2009, 38, 395-404.

26 J. Isaac, S. Ganguly and A. Ghosh, Eur. J. Pharm. Biopharm., 2016, 101, 43-52.

27 P. Lepek, W. Sawicki, K. Wlodarski, Z. Wojnarowska, M. Paluch and L. Guzik, Eur. J. Pharm. Biopharm., 2013, 83, 114-121.

28 R. E. Dinnebier, P. Sieger, H. Nar, K. Shankland and W. I. F. David, J. Pharm. Sci., 2000, 89, 1465-1479.

29 R. Dukeck, P. Sieger and P. Karmwar, Eur. J. Pharm. Sci., 2013, 49, 723-731.

30 R. J. Boghra, P. C. Kothawade, V. S. Belgamwar, P. P. Nerkar, A. R. Tekade and S. J. Surana, Chem. Pharm. Bull., 2011, 59, 438-441.

31 N. Boutonnet-Fagegaltier, J. Menegotto, A. Lamure, H. Duplaa, A. Caron, C. Lacabanne and M. Bauer, J. Pharm. Sci., 2002, 91, 1548-1560.

32 G. Chawla and A. K. Bansal, Sci. Pharm., 2009, 77, 695-709.

33 G. Chawla and A. K. Bansal, Eur. J. Pharm. Sci., 2007, 32, 4557.

34 G. Chawla and A. K. Bansal, Acta Pharm., 2008, 58, 257-274. 35 Z. Bocskei, K. Simon, R. Rao, A. Caron, C. A. Rodger and M. Bauer, Acta Crystallogr., Sect. C: Cryst. Struct. Commun., 1998, 54, 808-810.

36 J. J. Moura Ramos, R. Taveira-Marques and H. P. Diogo, J. Pharm. Sci., 2004, 93, 1503-1507.

37 H. P. Diogo, M. T. Viciosa and J. J. Moura Ramos, Thermochim. Acta, 2016, 623, 29-35, DOI: 10.1016/ j.tca.2015.11.012.

38 G. Teyssedre and C. Lacabanne, J. Phys. D: Appl. Phys., 1995, 28, 1478-1487.

39 J. van Turnhout, Thermally stimulated discharge of polymer electrets, Elsevier, Amsterdam, 1975.

$40 \mathrm{R}$. Chen and Y. Kirsh, Analysis of Thermally Stimulated Processes, Pergamon Press, Oxford, 1981.

41 J. van Turnhout, Thermally stimulated discharge of electrets, in Electrets, ed. G. M. Sessler, Springer, Berlin, Heidelberg, 1987, pp. 81-215.

42 V. M. Gun'ko, V. I. Zarko, E. V. Goncharuk, L. S. Andriyko, V. V. Turov, Y. M. Nychiporuk, R. Leboda, J. SkubiszewskaZieba, A. L. Gabchak, V. D. Osovskii, Y. G. Ptushinskii, G. R. Yurchenko, O. A. Mishchuk, P. P. Gorbik, P. Pissis and J. P. Blitz, Adv. Colloid Interface Sci., 2007, 131, 1-89. 
43 B. B. Sauer, Thermally Stimulated Currents: Recent Developments in Characterisation and Analysis of Polymers, in Applications to Polymers and Plastics, ed. Cheng and Z. D. Stephen, Elsevier, Amsterdam, 2002, pp. 653-711.

44 A. Vassilikou-Dova and I. M. Kalogeras, in Thermal Analysis of Polymers: Fundamentals and Applications, ed. J. D. Menczel and R. B. Prime, John Wiley, Hoboken, New Jersey, 2009, ch. 6, pp. 497-613.

45 N. Boutonnet-Fagegaltier, A. Lamure, J. Menegotto, C. Lacabanne, A. Caron, H. Duplaa, and M. Bauer, in Thermal Analysis of Pharmaceuticals, ed. D. Q. M. Craig and M. Reading, CRC Press, Boca Raton, 2007, ch. 11, pp. 359382.

46 S. Barker and M. D. Antonijevic, in Solid state characterization of pharmaceuticals, ed. R. A. Storey and I. Ymén, Blackwell Publishing, Chichester, 2011, ch. 5, pp. 187-206.

47 C. F. Macrae, I. J. Bruno, J. A. Chisholm, P. R. Edgington, P. McCabe, E. Pidcock, L. Rodriguez-Monge, R. Taylor, J. van de Streek and P. A. Wood, J. Appl. Crystallogr., 2008, 41, 466-470.

48 N. R. Xivillé, L. T. Lorente and A. Kordikowski, Int. J. Pharm., 2012, 422, 271-279.
49 K. Adrjanowicz, K. Grzybowska, K. Kaminski, L. Hawelek, M. Paluch and D. Zakowiecki, Philos. Mag., 2011, 91, 19261948.

50 A. C. Montbazin, D. Chantreux, and C. Bouloumie, Process for the preparation of a tetrazole derivative in two crystalline forms and novel the crystalline forms thereof, US Pat., 5, 629, 331, 1997.

51 R. Hilfiker, Polymorphism in the pharmaceutical industry, Wiley-VCH, Weinheim, 2006.

52 R. A. Carlton, in Pharmaceutical microscopy, Springer, New York, 2011, ch. 8, pp. 213-246.

53 C. T. Moynihan, A. J. Easteal, J. Wilder and J. Tucker, J. Phys. Chem., 1974, 78, 2673-2677.

54 J. D. Menczel, L. Judovits, R. B. Prime, H. E. Bair, M. Reading and S. Swier, in Thermal Analysis of Polymers: Fundamentals and Applications, ed. J. D. Menczel, Wiley, Hoboken, New Jersey, 2009, ch. 2, pp. 7-239.

55 R. Svoboda and J. Málek, J. Non-Cryst. Solids, 2013, 378, 186195.

56 R. Svoboda, J. Therm. Anal. Calorim., 2014, 118, 1721-1732.

57 K. J. Crowley and G. Zografi, Thermochim. Acta, 2001, 380, 7993.

58 C. A. Angell, J. Non-Cryst. Solids, 1991, 131-133, 13-31.

59 R. Böhmer, K. L. Ngai, C. A. Angell and D. J. Plazek, J. Chem. Phys., 1993, 99, 4201-4209. 